\title{
The Impact of Calls to the Processor on a Server PABX
}

\author{
Ndaohialy Manda-Vy Ravonimanantsoa, Andry Auguste Randriamitantsoa, and Malalatiana Hajasoa \\ Ramafiarisona
}

\begin{abstract}
The main theme of this paper is to define the relationship between the number of calls made in an Asterisk server and use of the processor in this server as the processor is one of the major hardware resources. Subsequently, we derived the formulation for the generalization of this relationship or a mathematical model will be obtained. In conclusion of this article, the results of the mathematical model developed are compared with results obtained in the experiment from the graph produced with scilab.
\end{abstract}

\section{Index Terms-Asterisk, Sip, CPU, VoIP.}

\section{INTRODUCTION}

Research by Pablo Montoro and Eduardo casilari [1] lead us to the realization of this paper. The results of their research enabled us to even another aspect of IP telephony especially in the use of server processors. Obviously the Voice over Internet Protocol (VoIP) technology has become the most used Internet in terms of communication. The use of various protocol standards by manufacturers shows the orientation of research towards this technology phone. In addition we are now witnessing a convergence towards "everything over IP". An advance in research around the new information technology and communication specifies those trend and utilities said open sources are a remarkable progress in the technology and especially in the transmission of voice over IP network. The communication circuit to packet communication it is still a current model [2] [3] [4].

The existence of many standard protocol level leads many researchers worked on the difference between the protocol [1],SIP (Session Initiation Protocol) is one of those protocols and open source alone accounts for many many documentation and research [5] [6] [7] [8] [9].

Our works is therefore in the study and analysis of the existence of a relationship between the numbers of calls and use the server's CPU.

Using a server PABX (Private Automatic Branch eXchange) Asterisk is also essential for this kind of research for his license [7] its easy installation and its many features [10] [11] [12].

Our work is structured as follows: Section 2 summarizes the main results of research already done and we made based on our research and the results of some previous work that is to say the state of the art. Section 3 describes how we used to arrive at our conclusion. The results are commented in Section 4, while Section 5 contains the main model and

Manuscript received March 17, 2012; revised May 8, 2012.

Ndaohialy Manda-Vy Ravonimanantsoa, Andry Auguste Randriamitantsoa, and Malalatiana Hajasoa Ramafiarisona are with the ESPA, University of Antananarivo (e-mail: ndaohialy@blueline.mg). formula that we derived from our research.

\section{STATE OF THE ART}

In the article "a comparative study of standard Voip with asterisk", the authors do a lot of different research-based protocol used in VoIP. This experiment is as follows:

The block diagram of the system deployed for the study, shown in Fig. 1, comprises three components: an Asterisk server (the unit under observation), a call generator (for calls to be sent to asterisk), and a Server Monitor (that will monitor the Asterisk server). Fig. 2 depicts the interconnection of these three elements: the call generator and the Asterisk server are connected by two different Ethernet interfaces (eth0 and eth1). The calls will initially be transmitted via eth1 link, which supports the VoIP flow. The monitoring equipment is connected to the same switch as the eth0 link the other two units. For the Asterisk server, they installed a Debian GNU Linux system on a Pentium 4 (2'4 GHz) with $1 \mathrm{~GB}$ of RAM memory. Asterisk 1.4 has been installed from the Debian packages, using a standard configuration [2]

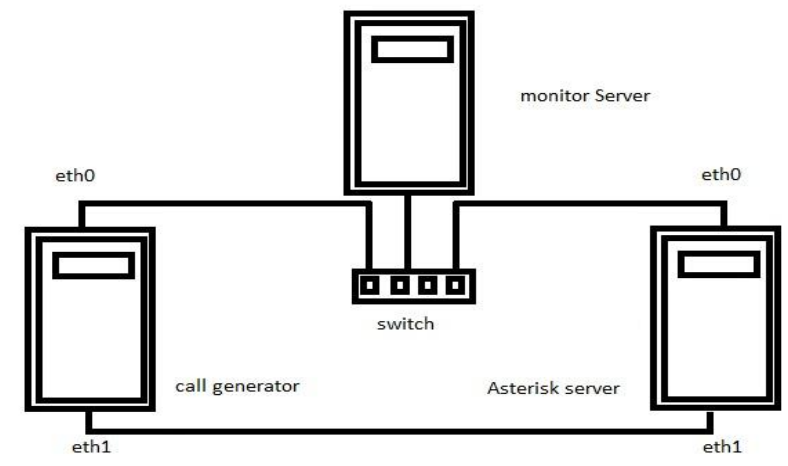

Fig. 1. Test procedure

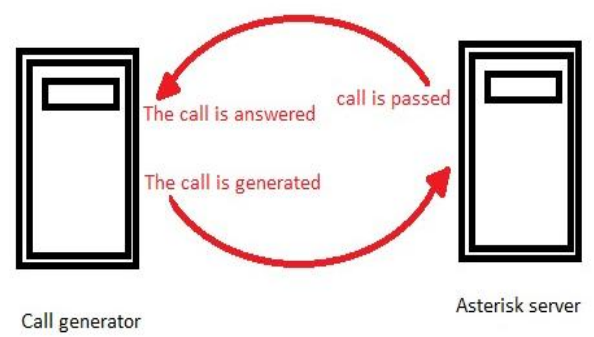

Fig. 2. Call generator

For the result we took only the result that uses the G711 codec and SIP [13] and this result is in Table 1

TABLE I: FIRST RESULT

\begin{tabular}{|l|l|}
\hline Call & CPU Utilization $(\%)$ \\
\hline 10 & 2,36 \\
\hline 20 & 4,64 \\
\hline 30 & 7,12 \\
\hline
\end{tabular}




\section{DESCRIPTION CALCULATES CORRELATION}

Study the correlation between two or more random variables or numerical statistics, this study is the intensity of the connection that may exist between these variables. In our case we have three four variable ie the number of calls, CPU usage, memory usage and finally the use of bandwidth. The connection is an affine relation sought.

A measure of this correlation is obtained by calculating the linear correlation coefficient. This coefficient is the ratio of their covariance and nonzero product of their standard deviations. The correlation coefficient is between -1 and 1 .

$$
\mathrm{R}(\mathrm{x}, \mathrm{y})=\frac{\operatorname{cov}(\mathrm{x}, \mathrm{y})}{\text { ecartype } \mathrm{x} * \text { ecartype } y}
$$

With $\operatorname{cov}(\mathrm{x}, \mathrm{y})=\frac{1}{\mathrm{~N}} \sum_{\mathrm{n}=1}^{\mathrm{n}} \mathrm{x}_{\mathrm{i}} \mathrm{y}_{\mathrm{i}}-\overline{\mathrm{xy}}$

If $R(x, y) \simeq \pm 1$ then $x$ and $y$ are correlated

if $\mathrm{R}(\mathrm{x}, \mathrm{y}) \simeq 0$ then $\mathrm{x}, \mathrm{y}$ aren't correlated,

In our case we took as a variable

$x=$ number of calls

$y=$ CPU usage

So we have

$R(x, y)=0,99970589$

\section{A. Comments on Results}

In our results we have found a correlation between the number of calls and use of the processor because

$R(x, y) \simeq 0.99970589$

\section{B. Mathematical Model}

To do so we took the method of least squares to find out if there is a correlation between two variable there will be an adjustment as a right and that right has the form $y=a x+b$ with

and

$$
a=\frac{\sum x y-n \bar{x}-\bar{y}}{\sum x^{2}-n \bar{x}^{2}}
$$

$$
b=\bar{y}-a \bar{x}
$$

Thus we have obtained

$$
\begin{aligned}
& Y=0,238 x-0,05333 \\
& y=f(x)
\end{aligned}
$$

So to $2.4 \mathrm{GHz}$ clock frequency, we have 4286 customers. And after verification we obtained the following table II

TABLE I: RESULT OF F(X)

\begin{tabular}{|l|l|}
\hline call & CPU Utilization (\%) \\
\hline 10 & 2,32667 \\
\hline 20 & 4,70667 \\
\hline 30 & 7,08 \\
\hline
\end{tabular}

From this table we could see that the result of our model resembles approximately the same reality this we have deduced that can calculate the size of the power of our memory and CPU utilization of bandwidth a given Asterisk server if you know the number of client and back that is to say, knowing the capacity of our server hardware we can deduce the number of possible customer you can assign to this server.

We compared the graphs of these two results that we obtained by the call generator and the one obtained by our equation with the software scilab and this graph shows a similarity of the two results.

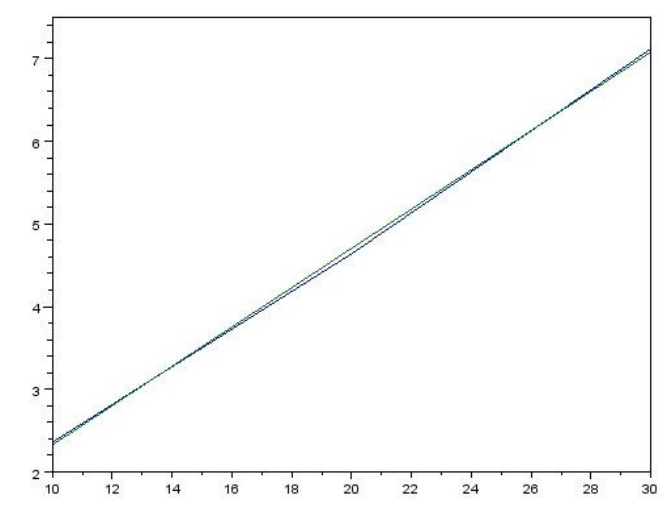

Fig. 3. Graphical comparison of results

The graph in green represents the first result of the experiment and the graph in blue that of the equation $y=a x+$ b.

\section{CONCLUSION}

We calculate the correlation we found that obviously there was a relationship between the number of call and CPU usage, we can say therefore is that the close relationship between the number of calls made and the percentage of CPU usage. This relationship leads us to infer that if we continue to increase the number of calls could lead to a limit so that this limit represents the physical capacity of our asterisk server.

Using a low hardware limits the number of users to an Asterisk server. However, we found that the software point of view, seen Asterisk is free software; this constraint can be solved at the software.

The question that arises is: should it improved the performance of a processor or improved Asterisk PBX to increase the number of users of an asterisk server?

\section{REFERENCES}

[1] P. Montoro and E. Casilari, "A comparative study of VoIP Standards with Asterisk," Forth international conference On Digital telecommunication, 2009.

[2] J. V. Meggelen, J. Smith, and L. Madsen, Asterisk - "The Future of Telephony," 2nd ed., Sebastopol (USA): O'Reilly Media, Inc., Aug. 2007.

[3] V. N. G. J. Soares, P. A. C. Neves, and J. J. P. Rodrigues, "Past, Present and Future of IP Telephony," Proc. of Communication Theory, Reliability, and Quality of Service (CTRQ'08), Bucharest (Romania), Jul. 2008, pp. 19-24.

[4] H. M. Chong, and H. S. Matthews, "Comparative Analysis of Traditional Telephone and Voice-over-Internet protocol (VoIP) Systems", Proc. of IEEE International Symposium on Electronics and the Environment, Scottdale (AZ USA), May 2004, pp.106-111.

[5] B. Chatras and S. Garcin, "Service drivers for selecting VoIP protocols", Proc. of Telecommunications Network Strategy and Planning Symposium, Vienna (Austria), Jun. 2004, pp. 131-136.

[6] Basicevic, M. Popovic, and D. Kukolj, "Comparison of SIP and H.323 Protocols," Proc. of the Third International Conference on Digital Telecommunications (ICDT'08), Bucharest (Romania), Jul. 2008, pp.162-167.

[7] T. Abbasi, S. Prasad, N. Seddigh, and I. Lambadaris, "A comparative study of the SIP and IAX VoIP protocols," Proc. of Canadian Conference on Electrical and Computer Engineering, Saskatoon (Saskatchewan Canada), May 2005, pp. 179-183.

[8] M. E. Nasr and S. A. Napoleon, "On improving voice quality degraded by packet loss in data networks," Proc. of the Twenty-Second National 
Radio Science Conference (NRSC 2005), Cairo (Egypt), Mar. 2005, pp. 465-472.

[9] L. Deri, "Open Source VoIP Traffic Monitoring," Proc. of System Administration and Network Engineering (SANE 2006), Delft (The Netherlands), May. 2006.

[10] G. Zhang, M. Hillenbrand, and P. Muller, "Facilitating the Interoperability among Different VoIP Protocols with VoIP Web Services," Proc. of First International Conference on Distributed Frameworks for Multimedia Applications (DFMA'05), Besançon(France), Feb. 2005, pp. 39-44.

[11] M. Z. Alam, S. Bose, M. M. Rahman, and M. A. Al-Mumin, "Small Office PBX Using Voice Over Internet Protocol (VOIP)," Proc. of
IEEE The 9th International Conference on Advanced Communication Technology (ICACT 2007), vol.3, Gangwon-Do(Korea), Feb. 2007, pp. 1618-1622.

[12] Tressel and J. Keller, "A System for Secure IP Telephone Conferences," Proc. of the Fifth IEEE International Symposium on Network Computing and Applications, Cambridge (Massachusetts), Jul. 2006, pp. 231-234.

[13] A. Gorti, "A fault tolerant VoIP implementation based on open standards," Proc. of Sixth European Dependable Computing Conference (EDCC'06), Coimbra (Portugal), Oct. 2006, pp. 35-38. 